\title{
Can FDG-PET imaging play a role in guiding indications to endovascular treatments in patients presenting acute aortic syndromes?
}

\author{
Michael Soussan, MD, PhD, ${ }^{\mathrm{a}}$ and Fabien Hyafil, $M D, \mathrm{PhD}^{\mathrm{b}}$ \\ a Department of Nuclear Medicine, Hopital Avicenne, Assistance Publique - Hôpitaux de Paris, \\ Université Paris 13, Bobigny, France \\ b Department of Nuclear Medicine, Centre Hospitalier Universitaire Bichat, Assistance Publique - \\ Hôpitaux de Paris, Inserm 1148, DHU FIRE, Université Paris Diderot, Paris, France
}

Received Sep 15, 2017; accepted Sep 16, 2017

doi:10.1007/s12350-017-1077-7

\section{See related article, pp. 633-641}

\section{MANAGEMENT OF ACUTE AORTIC SYNDROMES}

Acute aortic syndrome (AAS) groups are life-threatening conditions affecting the aortic wall. AAS are characterized by a disruption of the medial layer of the aorta and consist of acute aortic dissection (AD), intramural hematoma (IMH), and penetrating aortic ulcer (PAU). ${ }^{1}$ Aortic dissection is the most common form of AAS, IMH, and PAU representing only $10 \%$ of AAS. Acute aortic dissection and intramural hematoma share similar clinical features and complications, but have different pathophysiological mechanisms. ${ }^{2}$ Acute aortic dissection is characterized by the presence of an entry tear that typically occurs spontaneously, whereas intramural hematoma is caused by the rupture of vasa vasorum in the media. IMH can, however, progress to dissection if the intimal layer ruptures. PAU are mostly caused by the ulceration of complex atherosclerotic plaques. Patients with $\mathrm{AD}$ of the ascending thoracic aorta and with $\mathrm{PAU}^{3}$ have poor spontaneous prognosis and require urgent open surgery. In patients with IMH, about one third of the patients evolve towards complete aortic dissection. In patients with AAS involving the aortic arch and the descending thoracic aorta, a multidisciplinary approach is

Reprint requests: Michael Soussan, MD, PhD, Department of Nuclear Medicine, Hopital Avicenne, Assistance Publique - Hôpitaux de Paris, Université Paris 13, Bobigny, France; michael.soussan@avc.aphp.fr

J Nucl Cardiol 2019;26:642-4.

$1071-3581 / \$ 34.00$

Copyright (c) 2017 American Society of Nuclear Cardiology. required to select the optimal treatment for each patient among open surgery, endovascular intervention, or medical management. Open surgery involving the descending thoracic aorta is complex and can be complicated by paraplegia or paraparesis in case of occlusion of the Adamkiewicz artery perfusing the spinal cord that can occur in around 5\% of patients. ${ }^{4}$ In the past 10 years, significant progresses have been made in endovascular approaches of AAS. Dedicated vascular prosthesis has been developed for thoracic endovascular aortic repair (TEVAR) and are associated with less complication than open surgery. TEVAR has become the treatment of choice in $\mathrm{AD}$ of the descending aorta with malperfusion syndrome with a technical success rate of $90 \% .^{5}$ However, complications such as endoleak, aneurysms of the distal aorta, or continued false lumen perfusion, have been described in 5-10\% of patients. ${ }^{5}$ TEVAR appears also as a safe technique for complicated type B IMH, providing very good long-term results. ${ }^{6}$ However, TEVAR can be complicated by retrograde $\mathrm{AD}$ or pseudoaneurysm in patients with IMH. ${ }^{7}$ The search for factors predictive of poor outcome is thus important to select the patients with AAS that will benefit the most from interventional endovascular treatments at the expense of complications of prosthesis in a minority of them.

\section{WHAT IS THE RATIONALE FOR USING FDG-PET IN AAS?}

FDG-PET imaging offers to assess noninvasively the degree of inflammation in the vascular wall. ${ }^{8-10}$ In atherosclerotic plaques, the intensity of FDG uptake is related to the degree of macrophage infiltration. In acute aortic syndrome, the FDG signal might rather originate from an inflammatory reaction in the adventitia. The 
adventitia is the outer layer of the aorta that maintains the integrity of the vessel in case of vascular lesion; thanks to its high fibrotic content. The infiltration of this layer by inflammatory cells might reflect both the depth of the lesion into the vascular wall but might also play an active role in the weakening of this structure through local secretion of proteolytic enzymes by inflammatory cells. ${ }^{11}$ Few studies suggest that the intensity of FDG uptake might help to identify patients with poor outcome in acute aortic syndrome. In patients with abdominal aortic aneurysm (AAA), Reeps et al. found that the intensity of FDG uptake in AAA was associated with progression and correlates with the number of macrophage and T-cell, higher local MMP tissue activities, and extracellular matrix degradation. ${ }^{12,13}$ Similarly, Kuehl et al. showed, in 33 patients with AAS, that vessel wall inflammation depicted by FDG-PET was found in one third of the patients. ${ }^{14}$ During the follow-up period, 9 of 11 PET-positive patients $(82 \%)$ showed progression of AAS, whereas $55 \%$ of PET-negative patients showed stable disease or regression. Furthermore, Sakalisahan et al. confirmed in a series of 23 patients with type $\mathrm{B}$ dissection that increased FDG uptake has an incremental prognostic value in association to the maximal diameter of the aortic dissection, partial thrombosis of the false channel, and plasma markers of coagulation and fibrinolysis. ${ }^{15}$

In this issue of JNC, Yang and colleagues provide additional data on the potential role of FDG-PET in acute aortic syndrome (AAS). They included 34 patients with acute type B intramural hematoma (IMH) who underwent PET/CT within 14 days from the onset of symptoms, to evaluate the association of FDG uptake with the occurrence of adverse aortic events during follow-up : conversion to TEVAR, development of AD, enlargement of newly developed ulcer-like projection (ULP: sites of intimal disruption), and aortic enlargement. They found that the maximal standardized uptake values (SUVmax) was significantly higher in patients with adverse aortic events $(4.3 \pm 0.6$ vs. $3.7 \pm 1.0, P=0.02)$. A subgroup analysis, in patients with IMH and ULP $(n=25)$, also showed an association between the intensity of FDG uptake (SUVmax and target to blood ratio) at baseline and the occurrence of adverse aortic events during follow-up. Based on these results, the Authors suggest that patients with IMH + ULP and high-FDG uptake should be closely monitored. A threshold of TBR (target to blood ratio) $>1.5$ for predicting adverse aortic events, with a sensitivity at $73 \%$ and a specificity at $80 \%$ is also proposed. The work presented in this issue of JNC that studied more specifically on patients with IMH and PAU is thus in line with the results of former studies demonstrating the prognostic value of FDG-PET imaging in patients with AAS.

\section{CHALLENGES FOR IMPLEMENTING FDG-PET AS A BIOMARKER IN AAS}

Molecular imaging can provide complementary information to morphological imaging. The definition of reproducible and solid metrics is the key for the implementation of the results of imaging in clinical management. One important limitation of the study of Yang et al. is the methodology used for the imaging of the vessel wall with PET. In this study, PET acquisitions were acquired after injection of high activities of FDG to patients (7 MBq/kg), at early time points after injection (1 hour) and with relatively short acquisition times $(2 \mathrm{mn} /$ bed position) in contrast to the current recommendations for vascular imaging. ${ }^{16}$ In fact, these choices might significantly affect the validity of the quantitative measurements that have been performed in this work. Measurements performed in the vascular wall are very sensitive to parameters selected for image acquisition and reconstruction as well as to partial-volume effects, in particular in relation to the residual blood signal. ${ }^{17}$ In this study, the mean SUV of blood was 2.5. This high residual signal of blood might have impacted the max. SUV measured in lesions through the penetration of blood in PAU and partial-volume effects. Partial-volume effect can also lead to an underestimation of the activity of small structures, typically whenever the lesion size is less than 3 times the spatial resolution of the system. This effect is caused by an image blurring introduced by the finite spatial resolution of the imaging system and by the tissue fraction effect (caused by image sampling). ${ }^{18}$ Partial volume effect particularly affects signal quantification in the vessel wall, thinner than $5 \mathrm{~mm}$. This raises the question of the reproducibility of PET measurements and the generalization of the thresholds calculated in monocentric studies, as well as the performance of PET imaging for assessing small anatomical structures. The results of this study underscore the need to agree on standardized PET acquisition protocols and metrics for vascular imaging in order to generate robust and solid parameters that can be extracted from molecular imaging data and have the best chances to be implemented into guide clinical decision.

\section{Disclosure}

Michael Soussan and Fabien Hyafil have nothing to disclose.

\section{References}

1. Clough RE, Nienaber CA. Management of acute aortic syndrome. Nat Rev Cardiol. 2015;12:103-14. doi:10.1038/nrcardio.2014.203. 
2. Mussa FF, Horton JD, Moridzadeh R, et al. Acute aortic dissection and intramural hematoma: A systematic review. JAMA. 2016; 316:754-63. doi:10.1001/jama.2016.10026.

3. Evangelista A, Mukherjee D, Mehta RH, et al. Acute intramural hematoma of the aorta: a mystery in evolution. Circulation. 2005;111:1063-70. doi:10.1161/01.CIR.0000156444.26393.80.

4. Coselli JS, Bozinovski J, LeMaire SA. Open surgical repair of 2286 thoracoabdominal aortic aneurysms. Ann Thorac Surg. 2007;83:S862-4-892. doi:10.1016/j.athoracsur.2006.10.088.

5. Thrumurthy SG, Karthikesalingam A, Patterson BO, et al. A systematic review of mid-term outcomes of thoracic endovascular repair (TEVAR) of chronic type B aortic dissection. Eur J Vasc Endovasc Surg Off J Eur Soc Vasc Surg. 2011;42:632-47. doi: 10.1016/j.ejvs.2011.08.009.

6. Felisaz A, Dufranc J, Heyndrickx M, et al. Midterm results of type B intramural hematoma endovascular treatment. Ann Vasc Surg. 2015;29:898-904. doi:10.1016/j.avsg.2014.12.024.

7. Sueyoshi E, Nagayama H, Hashizume K, et al. Computed tomography evaluation of aortic remodeling after endovascular treatment for complicated ulcer-like projection in patients with type B aortic intramural hematoma. J Vasc Surg. 2014;59:693-9. doi:10.1016/j.jvs.2013.08.100.

8. Klink A, Hyafil F, Rudd J, et al. Diagnostic and therapeutic strategies for small abdominal aortic aneurysms. Nat Rev Cardiol. 2011;8:338-47. doi:10.1038/nrcardio.2011.1.

9. Soussan M, Nicolas P, Schramm C, et al. Management of largevessel vasculitis with FDG-PET: A systematic literature review and meta-analysis. Medicine (Baltimore). 2015;94:e622. doi: 10.1097/MD.0000000000000622.

10. Hommada M, Mekinian A, Brillet P-Y, et al. Aortitis in giant cell arteritis: diagnosis with FDG PET/CT and agreement with CT angiography. Autoimmun Rev. 2017;. doi:10.1016/j.autrev.2017. 09.008 .

11. Courtois A, Nusgens BV, Hustinx R, et al. 18F-FDG uptake assessed by PET/CT in abdominal aortic aneurysms is associated with cellular and molecular alterations prefacing wall deterioration and rupture. J Nucl Med Off Publ Soc Nucl Med. 2013;54:1740-7. doi:10.2967/jnumed.112.115873.

12. Reeps C, Bundschuh RA, Pellisek J, et al. Quantitative assessment of glucose metabolism in the vessel wall of abdominal aortic aneurysms: correlation with histology and role of partial volume correction. Int $\mathbf{J}$ Cardiovasc Imaging. 2013;29:505-12. doi: 10.1007/s10554-012-0090-9.

13. Reeps C, Essler M, Pelisek J, et al. Increased 18F-fluorodeoxyglucose uptake in abdominal aortic aneurysms in positron emission/computed tomography is associated with inflammation, aortic wall instability, and acute symptoms. J Vasc Surg 2008;48:417-423; discussion 424. doi: 10.1016/j.jvs.2008.03.059

14. Kuehl H, Eggebrecht H, Boes T, et al. Detection of inflammation in patients with acute aortic syndrome: Comparison of. Heart $\mathrm{Br}$ Card Soc. 2008;94:1472-7. doi:10.1136/hrt.2007.127282.

15. Sakalihasan N, Nienaber CA, Hustinx R, et al. (Tissue PET) Vascular metabolic imaging and peripheral plasma biomarkers in the evolution of chronic aortic dissections. Eur Heart J Cardiovasc Imaging. 2015;16:626-33. doi:10.1093/ehjci/jeu283.

16. Bucerius J, Hyafil F, Verberne HJ, et al. Position paper of the Cardiovascular Committee of the European Association of Nuclear Medicine (EANM) on PET imaging of atherosclerosis. Eur J Nucl Med Mol Imaging. 2016;43:780-92. doi:10.1007/s00259-0153259-3.

17. Huet P, Burg S, Le Guludec D, et al. Variability and uncertainty of 18 F-FDG PET imaging protocols for assessing inflammation in atherosclerosis: suggestions for improvement. J Nucl Med Off Publ Soc Nucl Med. 2015;56:552-9. doi:10.2967/jnumed.114. 142596.

18. Soret M, Bacharach SL, Buvat I. Partial-volume effect in PET tumor imaging. J Nucl Med. 2007;48:932-45. doi:10.2967/ jnumed.106.035774. 